\title{
PRELIMINARY INVESTIGATION OF QUANTITATIVE SPECTRAL CLASSIFICATION BY MEANS OF OBJECTIVE-PRISM SPECTRA OBTAINED WITH THE BROERFELDE SCHMIDT TELESCOPE
}

\author{
J. V. CLAUSEN \\ Copenhagen University Observatory, Brærfelde, Denmark \\ (Read by R. M. West)
}

\begin{abstract}
Different methods, used for measuring the strength of hydrogen lines in objective-prism spectra, are discussed and the parameters are compared with the photoelectric $\beta$-index. It is found that for A5V-G1V stars $\beta$ can be given with a mean error of $t 0$ m.020 (one spectrum).
\end{abstract}

\section{Introduction}

The object of this paper is to present some results from an examination of objectiveprism spectra obtained with the Brœrfelde Schmidt-telescope. The program was carried out in order to get an impression of to what extent these spectra can be used for quantitative spectral classification. Different methods, used for measuring the strength of hydrogen lines in spectra of A5V-G1V stars, are discussed and the parameters are compared with the photoelectric $\beta$-index.

\section{The Telescope}

The 50/77/150-cm Brœrfelde Schmidt-telescope was constructed with the principal purpose to obtain objective-prism spectra for accurate spectral classification. The instrument is equipped with a $15^{\circ}$ objective-prism made of $\mathrm{F} 2$ glass, giving a dispersion of $102 \AA \mathrm{mm}^{-1}$ at $\mathrm{H} \gamma$, and the photographic magnitude-limit is about $9 \mathrm{~m} .5$. The diameter of the field covered by the telescope is 5.3 .

The relatively short focal length ensures that, under normal conditions, i.e. seeing better than $3^{\prime \prime}$, the resolution of the spectra is plate-limited.

Generally, spectra obtained so far have indeed turned out to be of good quality.

\section{The Investigation}

Spectra obtained on 21 Kodak IIa-D plates covering 4 overlapping fields in the Hyades have been examined. The plates were taken on three different nights. Exposure times of 150 (telescope diaphragmed to $25 \%$-opening), 225 and $600 \mathrm{~s}$ were used in order to get well exposed spectra over the B magnitude-range $5^{\mathrm{m}}-9^{\mathrm{m}}$. The spectra were widened to $0.30 \mathrm{~mm}$.

159 spectra of 43 Hyades main-sequence stars covering the MK-type range A5VG1V were selected for the investigation. 
The spectra were recorded on the digitized microphotometer at the Copenhagen University Observatory, described by West (1972). The slit-width was $25 \mu$ corresponding to $2.5 \AA$ at $\mathrm{H} \gamma$ and the distance between the transmission-readings punched on paper tape was $10 \mu$. The plate-speed was $200 \mu \mathrm{s}^{-1}$. The dark current and fog transmission were read before and after each reading of a spectrum.

The photographic calibration was performed by using existing $U B V$ photometry (Johnson and Knuckles, 1955), uvby photometry (Crawford and Perry, 1966) and spectrophotometry (Oke and Conti, 1966) for Hyades-stars. The calibration curves could be well represented by the Baker-formula $\log I=B_{1} \cdot \log (1 / T-1)+B_{2}$, where $I$ is the intensity, $T$ the transmission, $B_{1}$ and $B_{2}$ the constants to be determined. The variation of $B_{1}$ with wavelength was taken into account and calibration curves were established separately for each plate.

Even for moderately high-dispersion objective-prism spectra the only parameter normally used for measuring line-strength is the line-depth. The main reason for this is that the line-depth is easily derived from the spectral registrations, whereas it used to be very time-consuming to derive other parameters which might be more accurately reproducable, at least for the stronger lines. This limitation is easily removed to-day when the output from the microphotometer can be fed directly into an electronic computer as is done in the present case.

Three different parameters describing the strength of each of the lines $\mathrm{H} \beta, \mathrm{H} \gamma$ and $\mathrm{H} \delta$ were computed.

(1) 'line-depth' $d$ in percentage of continuum intensity. Quasi-continua varying linearly with wavelength were used.

(2) 'equivalent width' $w$. Quasi-continua varying linearly with wavelength were used together with fixed line-limit wavelengths.

(3) $I(n, b)$-index defined by Furenlid (1971). $n$ and $b$ are the half-widths of the narrow and broad 'mathematical filters' centered on the line.

Several line-limit wavelengths were tried in computing $w$, as also different sets of $n$ and $b$ were tried in computing $I$. Lines with bottom-transmission outside the range covered by the photographic calibration were rejected.

The results from the different plates were compared. No systematic differences appeared to be present.

The mean value of the parameters are compared with the photoelectric $\beta$-index (Crawford and Perry, 1966). The relations between the parameters and $\beta$ were generally found to be linear for A5V-G1V stars, see Figure 1; the relations between $\beta$ and the $I$-indices for $\mathrm{H} \gamma$ and $\mathrm{H} \delta$ are, however, slightly curved for $\beta \leqslant 2.660$ corresponding to $\mathrm{F} 5 \mathrm{~V}$. The reason for this is certainly the greater influence on $I$ of lines near to $\mathrm{H} \gamma$ and $\mathrm{H} \delta$.

From Table $I$ it is seen that, for the Balmer lines (i.e. relatively strong lines), the $I$-index is superior to the two other parameters for measuring line-strength, especially when comparing with the line-depth. 


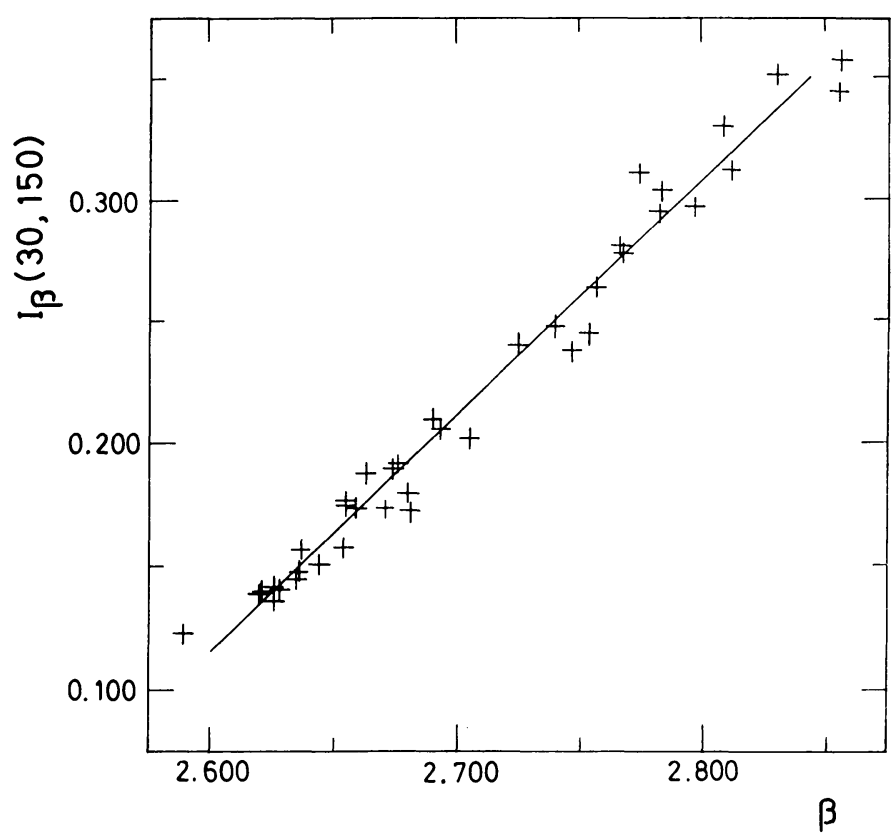

Fig. 1. $\beta$ vs $I_{\beta}(30,150)$ relation for A5V-G1V Hyades stars.

It is seen that for A5V-G1V stars $\beta$ can be given with a mean error of \pm 0.020 (one spectrum). For A5V-F5V stars, $\mathrm{H} \beta, \mathrm{H} \gamma$ and $\mathrm{H} \delta$ can be used with equal success. For F5V-G1V stars $\mathrm{H} \beta$ is preferable, the reason for this was already mentioned. The $\mathrm{H} \beta$-region is, unfortunately, not well recorded by the widely used IIa-O emulsion.

TABLE I

Calculated mean errors in $\beta$ (one spectrum)

$\begin{array}{lcc}\text { Parameter } & \begin{array}{c}\text { Internal m.e. } \\ \pm 0 \text { m.03 }\end{array} & \begin{array}{c}\text { External m.e. } \\ \pm 0.05\end{array} \\ d_{\beta} & 0.02 & 0.03 \\ w_{\beta} & 0.009 & 0.020 \\ I_{\beta}(30,150) & & \\ & & 0.06 \\ d_{\gamma} & 0.04 & 0.04 \\ w_{\gamma} & 0.02 & 0.020 \\ I_{\gamma}(30,120) & 0.010 & \left(\begin{array}{l}\beta \geqslant 2.660) \\ \end{array}\right. \\ I_{\gamma}(10,30) & 0.015 & 0.033 \\ & & (\beta \geqslant 2.660) \\ d_{\delta} & & 0.06 \\ w_{\delta} & 0.04 & 0.04 \\ I_{\delta}(30,105) & 0.02 & 0.019 \\ & 0.012 & (\beta \geqslant 2.660) \\ I_{\delta}(10,30) & & 0.028 \\ & 0.015 & (\beta \geqslant 2.660)\end{array}$


This preliminary investigation was restricted to A5V-G1V stars. We believe, however, that results of similar accuracy can be obtained for stars of all luminosity classes. Such an extension of the program is planned and will include also early-type objects. It should be recalled that for early-type stars, $\beta$ measures the luminosity, and for later A stars and for $\mathrm{F}$ stars $\beta$ is an indicator of spectral type.

The main purpose of our next program will be to establish schemes for quantitative 2- and 3-dimensional classification based on systematic examinations of the total lineinformation contained in objective-prism spectra of the present dispersion. The preliminary results discussed here indicate that the strength of the hydrogen lines measured by $I$-indices might be a valuable contribution to these schemes.

\section{References}

Crawford, D. L. and Perry, C. L.: 1966, Astron. J. 71, No. 3.

Furenlid, I.: 1971, Astron. Astrophys. 10, 321.

Johnson, H. L. and Knuckles, C. F.: 1955, Astrophys. J. 122, 209.

Oke, J. B. and Conti, P. S.: 1966, Astrophys. J. 143, 134.

West, R. M.: 1972, Bull. Abastumani Astrophys. Obs. 43, 109. 